\title{
Author Correction: PRMT1 promotes pancreatic cancer growth and predicts poor prognosis
}

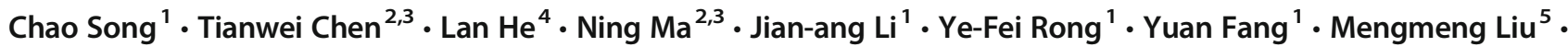 \\ Dong Xie $^{2,3} \cdot$ Wenhui Lou ${ }^{1}$
}

Published online: 2 December 2019

(C) International Society for Cellular Oncology 2019

\section{Author Correction: Cell Oncol}

https://doi.org/10.1007/s13402-019-00435-1

The authors would like to correct the following panels in Figures:

1) Figure 2c, the western blot band of GAPDH;

2) Figure 5a, the western blot band of GAPDH of Miacapa2 group.

The mistakes arose at assembling of the figures, and were missed by the authors during proofreading.
The correct panels are as follows:

1) Figure 2c:

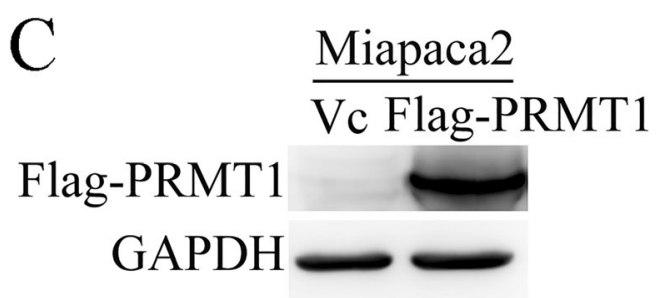

The online version of the original article can be found at https://doi.org/ 10.1007/s13402-019-00435-1

\footnotetext{
Dong Xie

dxie@sibs.ac.cn

$\triangle$ Wenhui Lou

lou.wenhui@zs-hospital.sh.cn

1 Department of Pancreatic Surgery, Zhongshan Hospital, Fudan University, Shanghai 200032, China

2 Laboratory of Molecular Oncology, Institute for Nutritional Sciences, Shanghai Institutes for Biological Sciences, Chinese Academy of Sciences, 320 Yue-Yang Rd., Shanghai 200031, China

3 University of the Chinese Academy of Sciences, Shanghai 200031, China

4 School of Life Science and Technology, Shanghai Tech University, Shanghai 201210, China

5 Department of Gastroenterology, Zhongshan Hospital, Fudan University, Shanghai 20032, China
} 
2) Figure 5a:

A

Capan-2

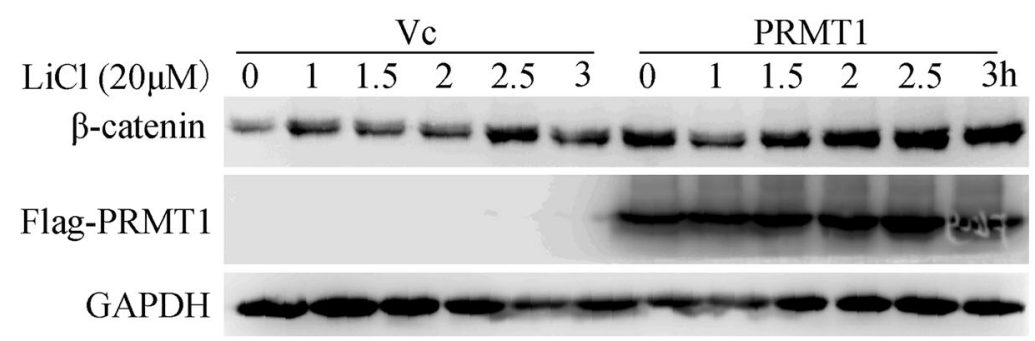

Miapaca2

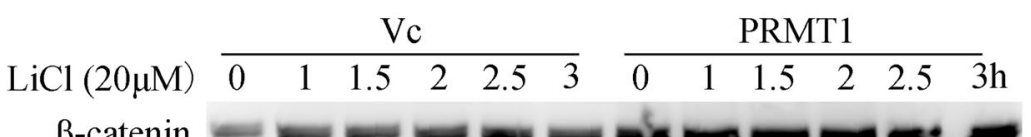

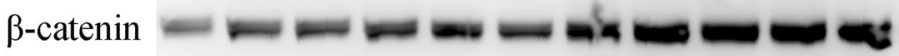

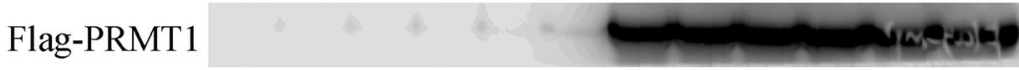

GAPDH

GAPDH served as loading controls in the figures, and the conclusions of the paper are not affected by this corrigendum. The remainder of Figure 2 and Figure 5 remains unchanged.
Publisher's note Springer Nature remains neutral with regard to jurisdictional claims in published maps and institutional affiliations. 